\title{
Performatividad: la teoría especial y la general ${ }^{1}$ Performativity: The Special and the General Theory
}

\author{
SONIA REVERTER-BAÑóN*
}

Universitat Jaume I, Castellón

Resumen. Si en Gender Trouble (1990) Butler presentaba una propuesta de la teoría de la performatividad de los actos de habla aplicada a la construcción del género, en su último libro, $N o$ tes towards a Performative Theory of Assembly (2015), articula una teoría de la performatividad aplicada a la acción colectiva de minorías o poblaciones que son estimadas como "desechables". El interés de la propuesta que presentamos es analizar cómo la teoría de la performatividad de género se ha ido ampliando a las formas de acción democrática; pasando así de ser una explicación centrada en las posibilidades identitarias de género a explicar las posibilidades para una vida vivible. Es lo que aquí llamamos la ampliación de la performatividad, del caso especial de género al caso general de una vida vivible.

Palabras clave: Judith Butler; performatividad; precariedad; asamblea; transformación social.
ABSTRACT. If in Gender Trouble (1990) Butler presented a proposal of the theory of performativity of speech acts applied to the construction of gender, in her last book, Notes towards a Performative Theory of Assembly (2015), she articulates a theory of performativity applied to collective and concerted action of minorities or populations that are estimated to be "disposable". The interest of the proposal that we present in this paper is to analyze how the theory of performativity of gender is now extended to the forms of democratic action; going from being a structure that explains the possibilities of gender to explain the possibilities for a livable life. It is what we call here the extension of performativity, from the special case of gender to the general case of a livable life.

Key words: Judith Butler; Performativity; Precarity; Precariousness; Assembly; Social Transformation.

${ }^{1}$ El presente trabajo ha sido posible gracias a la ayuda del Proyecto de investigación de I+D+I del Ministerio de Economía y Competitividad FFI2016-76753-C2-2P.

*reverter@fis.uji.es ORCID iD:http://orcid.org/0000-0002-9738-421X

Copyright: (C) 2017 CSIC. Este es un artículo de acceso abierto distribuido bajo los términos de la licencia de uso y distribución Creative Commons Attribution (CC-by) España 3.0 


\section{INTRODUCCIÓN}

El interés de la propuesta que presentamos es analizar cómo la teoría de la performatividad de género que Butler ofreció en Gender Trouble se amplía ahora a las formas de acción democrática. Ello explica el título del presente artículo, en el sentido de entender que la teoría performativa que Butler propone ha ido ampliándose a lo largo de los últimos 26 años; pasando así de ser una teoría que explica las posibilidades identitarias de género (de hacerlo y deshacerlo) a explicar las posibilidades para una vida vivible. Es lo que aquí llamamos la ampliación de la performatividad, del caso especial del género al caso general de cualquier constreñimiento normativo que posibilita o impide una vida vivible. Los conceptos de vulnerabilidad y precariedad serán el núcleo que permitirá esa ampliación de la performatividad, pues aunque como conceptos ya estaban en Gender Trouble, no habían sido tratados en profundidad en su relación con la pluralidad ${ }^{2}$.

El principal objetivo de Gender Trouble fue dejar claro, tal y como la autora ha declarado en obras posteriores, que la diferencia sexual puede ser contestada socialmente $(2004,212)$, y por ello su teoría de la performatividad es de sí una teoría política (Chambers \& Carver, 2008). Sin embargo, y como la misma Butler $(2004,213)$ reconoce, en su primer libro al ofrecer la metáfora del drag se permitió una interpretación que no dejaba ver con claridad cómo desde la parodia se puede ir a la política. De hecho Martha Nussbaum (1999) acusó a Butler de esto mismo en su crítico texto hacia la autora titulado "The Professor of Parody". Nussbaum (1999) culpaba a los "feminismos de estilo simbólico" de creer que emplear las palabras de manera subversiva y paródica, y escribir de forma oscura y abstracta, es hacer política feminista. Nussbaum interpretaba, como otras autoras han hecho, que Butler está proponiendo simplemente un feminismo autorreferencial que da la espalda a los problemas reales del mundo para dedicarse a la teoría abstracta, mostrando así poco compromiso público. ${ }^{3}$ Butler reconoce que "Probablemente escribí demasiado

${ }^{2}$ En el estudio de la teoría de la performatividad editado por Soley-Beltrán \& Sabsay (2012b), aunque centrado en la performatividad de género, las autoras ya afirman en la Introducción que se "insinúan posibles conexiones entre estas dos áreas de problematización" (2012a, 13), el género y la precariedad.

${ }^{3}$. Esta situación venía ya exasperada desde 1998, el año anterior, cuando Butler ganó el premio a la peor escritora (Bad Writing Contest) otorgado por la revista Philosophy and Literature. Butler (1999b) contestó inmediatamente defendiéndose en una columna en The New York Times, y en 2003 volvió a contestar con un escrito académico publicado en un libro editado que precisamente se titulaba Just being difficult? Antes de Nussbaum académicas feministas como Susan Gubar (1998) ya acusaron a Butler de escritura oscura. Para documentarse sobre esta contienda recomiendo Boynton (1999), Birkenstein (2010), y Pérez Navarro (2012) en el contexto español. 
rápido un texto cuyo futuro no anticipé en ese momento. ¿Por qué drag?"4 (2004, 213). El ejemplo del drag, admite la autora, no permitió entender la dimensión social y colectiva de la transformación política que conlleva (Butler, 2004, 216).

En una entrevista reciente (Zaharijevic, 2016) Butler reconoce que Gender Trouble no trató suficientemente el tema de las acciones sociales y políticas, y por ello no ofrece una manera adecuada de pensar las relaciones sociales que emergen de los movimientos colectivos. Ello posibilitó interpretar su propuesta de 1990 como una forma de individualismo, o incluso como un individualismo neo-liberal; algo que hizo que se sintiera en necesidad de repensar la "performatividad dentro de la pluralidad" (Zaharijevic, 2016, 106). Y es esto, precisamente, lo que Butler se propone hacer en el libro de 2015, Notes towards a Performative Theory of Assembly.

En el camino que va de Gender Trouble a Notes towards a Performative Theory of Assembly, y contando que hay 26 años entre estos dos libros, Butler reelabora su teoría de la performatividad. Como la misma autora recuerda en conversación con Sara Ahmed (Ahmed \& Butler, 2016, 482) en la segunda edición de Gender Trouble, de 1999, la autora ya reconoció que "la vida del texto ha excedido mis intenciones, y esto seguramente es en parte por el contexto cambiante de su recepción"5 (1999a, vii). El fenómeno global de los últimos años de movimientos de asambleas de ocupación de espacios públicos, junto con su insatisfacción por la deriva individualista que las interpretaciones de su primera obra tuvieron, ha sido lo que ha llevado a Butler a pensar en las formas de acción democrática. Butler afirma ahora, sin dar oportunidad de una nueva interpretación equivocada, que toda práctica política es esencialmente plural. Es decir, que congrega necesariamente una pluralidad de cuerpos, lo que ella llama en su última obra (2015b) "la asamblea pública de cuerpos". Ello conllevará, al mismo tiempo, la elaboración de una propuesta ética, algo que a decir de muchas autoras y autores faltaba en la obra de Butler (Lloyd, 2008, 2015a; Thiem, 2009; Rushing, 2010; Culberstone, 2013). Esta ausencia de una propuesta ética (o incluso política, como ya se encargó de señalar Benhabib en 1995) se subraya como una de las más importantes debilidades en el pensamiento de Butler; algo, sin embargo, que parece haber sido remediado en las últimas obras de la autora, muy especialmente a partir de Giving an Account of Oneself (2005). Es en esta obra que autoras como Magnus (2006), Mills (2007), Loizidou

${ }^{4}$ Traducción propia del texto "I probably wrote too quickly, a text whose future I did not anticipate at the time. Why drag?"

${ }^{5}$ Traducción propia de: "the life of the text has exceded my intentions, and this is surely in part because of the changing context of its reception". 
(2007), Zivi (2008), Burgos (2008), o Lloyd (2008, 2015a), nos permiten identificar lo que podemos denominar un "giro ético" en el pensamiento de Butler. La misma Butler reconoce en Giving an Account of Oneself $(2005,15)$ haber aceptado "demasiado rápidamente" la explicación de la subjetividad en Nietzsche a través del poder, sin tener en consideración que ello ya presupone la capacidad de rendir cuentas; capacidad que depende de relaciones éticas.

Interpretamos que este "giro ético" en la obra de Butler es claro en su última obra, Notes towards a Performative Theory of Assembly (2015b). Y el núcleo de ese giro es, de nuevo, el concepto de performatividad. La pregunta de quién cuenta como sujeto y quien no está vinculada a las diferentes formas performativas en que los sujetos son inteligibles y reconocidos. Las disrupciones performativas de aquellos que no cuentan como sujetos vinculan la performatividad con la precariedad, y ello nos pone frente a las puertas de las obligaciones éticas y políticas de aquellos (individuos y sistemas) que privan a la gente de tener una vida "vivible" (Butler, 2015b, 208). Y así, como disrupción performativa, es que podemos entender la congregación de cuerpos en asamblea, que frente a la precariedad de las vidas lucha por exigir una obligación ética y política: la de la convivencia. Proponemos, además, que si bien Butler confesó en el año 2000 en su entrevista con Connolly (Butler \& Connolly, 2000, 5) una "preocupación por el giro hacia la ética" porque éste se da normalmente desplazando a la política; en su propuesta en Notes towards a Performative Theory of Assembly (2015b) Butler articula una propuesta ética que no deja por ello de ser política. Tenemos que tener en cuenta que la misma Butler en "Ethical Ambivalence" $(2000,15)$ trató el tema de su resistencia previa a un "giro ético" y de la posterior "superación parcial" de esa resistencia.

\section{TEORÍA ESPECIAL: LA PERFORMATIVIDAD DE GÉNERO}

Butler considera que la teoría de la performatividad de género expuesta en Gender Trouble (1990) es "la principal contribución teórica del texto" (Ahmed \& Butler, 2016: 482). Veámos los principales rasgos de tal teoría.

La consecuencia más importante de la teoría de los actos de habla de John Austin (1962), revisada posteriormente por John Searle $(1969,1989)$, y corregida en parte por Derrida (1972), es que no hay posibilidad de separar el significado de la fuerza ilocucionaria; en definitiva de distinguir lo normativo o evaluativo por una parte y lo fáctico por otra. Esto conllevará la afirmación de que el lenguaje tiene la capacidad de llevar a término acciones; es decir, que al hablar actuamos. Como explica González Fisac $(2012,175)$ "el habla asume la inédita condición de acto". Esta capacidad de acción del lenguaje es lo que con- 
densa el término "performatividad" y es la que da juego a Butler en su libro de 1990 para explicar cómo esa capacidad nos permite entender mejor cómo se construye el género, proponiendo la llamada "teoría performativa de género".

Según ésta, que el género sea performativo quiere decir que no es estable o fijo, sino que es una característica identitaria creada y constituida a través de "una repetición estilizada de actos" $(1990,140)$. Butler hace extensiva esta naturaleza performativa al sexo. Para Butler el sexo no es una categoría que preexista al género, ni por tanto provee una fundamentación ontológica para géneros diferentes, o para significados de género binarios. Tanto el género como el sexo son discursivamente producidos por las normas y convenciones creadas y recreadas en el proceso de repetición/estabilización/legitimación/naturalización que forman la sociedad y su formación de discursos (el poder). El sexo queda, en la teoría feminista performativa, descentrado como sitio privilegiado para pensar y catalogar la identidad y la ética (Bell, 1999a, 9).

La pregunta que la tesis de Butler motivó dentro de los feminismos de los noventa fue: ¿Queda entonces el sujeto atrapado, a merced de ser construido enteramente de manera discursiva? Butler enseguida se encargó en algunos artículos (1991a), capítulos de libros (1991b, 1991c), entrevistas (1994) y en un libro posterior, Bodies that Matter (1993), de aclarar que para la teoría feminista performativa siempre hay posibilidad de resistencia y contestación, ya que la misma actividad de repetición de las normas abre también la posibilidad de subvertir estas normas. La agencia para Butler precisamente está en la variación que un sujeto puede producir al repetir (o no) activamente tales normas y convenciones. Según McNay $(1999,177)$ este punto está claro si vemos que la reiteración performativa de las normas simbólicas es necesaria para que éstas persistan; demostrando ello a la vez que tales normas no son naturales o "inevitables", y por tanto son potencialmente susceptibles de cambio. La pregunta entonces es isupone la performatividad un proceso voluntarista de performance, como algunas voces críticas interpretaron? Butler distingue, aunque no siempre con nitidez, como reconoce en el Prefacio a la edición del décimo aniversario de Gender Trouble (1999), entre performance y performatividad. La performance presupone un sujeto pre-existente, mientras que la performatividad reta la misma noción de sujeto $(1990,33)$. Butler se encargó más tarde, en su libro Bodies that Matter (1993), de aclarar mejor este controvertido punto. Butler aleja así su teoría de la performatividad de género de dos acusaciones contrapuestas: la de que se trata de un asunto meramente voluntarista y la de que el sujeto está tan determinado por las normas que no puede en realidad llevar a cabo ninguna resistencia ni transformación.

Las propuestas de Butler defendidas en sus libros de 1990 y 1993 generaron 
una corriente de investigación académica centrada en estudiar cómo la materialidad del cuerpo se construye de una manera discursiva. La teoría de Butler deconstruye en primer lugar la noción de cuerpo como algo "natural", dado, y previo a cualquier contexto. Para Butler el cuerpo se ha entendido y se explica como previo al signo, pero en realidad es al contrario: es el signo el que produce, constituye, construye, significa y resignifica el cuerpo. ¿Cómo explica esto? A través de la performatividad del lenguaje. Porque es precisamente esa cualidad del lenguaje la que permite la constitución del cuerpo de manera discursiva. El lenguaje no sólo refiere a una materialidad, sino que es la misma condición de que esa materialidad sea experimentada e interpretada. Esto ha conllevado significativas críticas a Butler, pues se entiende que se está negando la materialidad del cuerpo (Zita, 1992; Bordo, 1993; Segal, 1994; Grosz, 1995; Bigwood, 1998; Pearce, 2004) o incluso en peligro de caer en un idealismo lingüístico (Vasterling, 1999; Lloyd, 2007a; Loizidou, 2008a, 2008b; Jenkins, 2010).

De acuerdo con Butler, el cuerpo señala su presencia, y ello lo hace con una demanda en y para el lenguaje. Es en palabras de Butler "la demanda constitutiva que moviliza la acción psíquica desde el principio..." (1993, 67). La psique en realidad se reconfigura como un efecto de la interiorización de las normas sociales, y a la vez constituye, junto con el cuerpo (pues de hecho es cuerpo) el elemento principal de resistencia a las mismas normas. Aunque ese cuerpo no es un elemento extralingüístico, ni es ajeno al poder discursivo, como recalca repetidamente Burgos $(2008 ; 2012,118)$, no está cerrado, ni totalmente determinado como inteligible. Como Burgos $(2012,103)$ acertadamente nos dice: "El cuerpo materializado no se ajusta del todo a las normas. Queda un espacio, por la misma dinámica del proceso que implica una inestable materialización". Es decir, porque hay un cuerpo no inteligible (no cerrado normativamente del todo), con sus deseos no inteligibles, podemos experimentar la opresión de las normas. Precisamente este plus ontológico del cuerpo no interpretado, no inteligible, es lo que posibilita la consciencia de opresión y la búsqueda de experiencias alternativas ${ }^{6}$. Ello nos lleva a articular nuevas prácticas discursivas que posibilitan la contestación y transformación de las normas, es decir, el mismo proceso performativo.

¿Qué estrategias planteó Butler en los noventa para subvertir esas normas?

${ }^{6}$ Surge aquí un interesante debate sobre la ontología en el pensamiento de Butler. La autora $(1998,279)$ admite, en una entrevista, que es importante producir ontología como campo de contestación. La misma Butler (2015a) en un trabajo reciente explica cómo la relación entre el lenguaje y el cuerpo es un quiasma: "To say that the body is figured chiasmically is to say that the following logical relations hold simustaneously: the body is given through language, but is not, for that reason, reducible to language" (2015a, 21). Remito al importante trabajo de Elvira Burgos Díaz $(2008,2012)$ para esta cuestión 
Desde su planteamiento la norma más opresiva y más naturalizada, por ser la primera, que tenemos sobre el cuerpo es la heterosexualidad. Pues bien, un ejemplo contundente de transgredir esa norma es la transformación "drag", pues "reflexiona sobre la estructura imitativa por la cual el género hegemónico es en sí mismo producido, y discute la afirmación de la heterosexualidad como natural y original" (Butler, 1993, 125). Y aquí está precisamente la dimensión política de la identidad y el deseo. Ejemplos como el $d r a g$, aun con sus limitaciones y posteriores malinterpretaciones, como ya hemos indicado, sirvieron para comprender el alcance transformador de la performatividad de género.

El problema será, encontrar una noción "viable" de agencia, una noción que suponga un sujeto que no sea el mero portador del proceso de reiteración, sino también un posible participante en el proceso. Para Butler hay dos tipos de convenciones: las hegemónicas o dominantes, y las menores o subordinadas. Para ser sujeto necesariamente habré de seguir las convenciones hegemónicas, las condiciones no hegemónicas especifican las diferentes variaciones y formas de ser sujeto. Para ser descalificado como sujeto, para ser un "abyecto" (abject), bastará con no reiterar, de manera persistente con mis acciones y mis palabras, las convenciones hegemónicas. Butler cree que las exclusiones efectuadas por las convenciones hegemónicas son deshumanizadoras y violentas, y por ello debemos oponernos a ellas. Ahora bien, aunque no podemos aniquilar el poder mismo, sí podemos interceder en el proceso del poder, en sus mecanismos reiterativos, y por tanto empezar una nueva alternativa, un nuevo significado, que podrá (o no) tener en un futuro poder como norma. La ganancia que tenemos con esta acción es poder incluir una definición de sujeto más amplia, incluir a los "abyectos". Pero nunca podremos llegar a una inclusión total, por lo que el ideal de inclusividad radical es imposible (Butler, 1993, 193). ¿Para qué seguir luchando entonces? Para Butler la misma imposibilidad de llegar a esa idealización es la base de lo político, ya que es lo que motiva a una perpetua búsqueda y producción de posiciones políticas y sujetos políticos. Pero, ¿cómo motivarnos para luchar por algo que nunca alcanzaremos? Desde la teoría queda claro que una categoría lo es, precisamente porque incluye y excluye al mismo tiempo, así no puede haber una categoría que excluya todo o que incluya todo. La categoría de inclusividad total es imposible. Ahora bien, Butler admite que no todas las exclusiones son iguales, y que unas son más violentas que otras. Precisamente las más violentas serán aquellas producidas por las convenciones e interpretaciones hegemónicas. Así, retar estas convenciones supondrá reducir la violencia, aunque no reduzcamos a cero la exclusión.

Hemos dicho que la misma imposibilidad de la total inclusividad es la base de lo político. Para Butler la inclusividad total o radical supondría una reduc- 
ción de todas las diferencias, y ello comportaría un totalitarismo, es decir, la destrucción de la pluralidad, que no son más que diferencias constituidas por el habla y la acción. Por tanto, los mismos mecanismos de lo político y de la acción democrática necesitan mantener las diferencias, y por tanto, también cierta exclusión. Aun así, como ideal se mantiene la inclusividad radical. Entendemos que el programa político de Butler sería así la idea de una democracia radical que se base en pasar de una universalidad vacía con total inclusividad a una universalidad significativa, concreta. Necesitamos, pues, una distancia crítica entre el ideal de la democracia radical y el ideal del mundo perfecto o de la total inclusividad. Perder esta distancia crítica es caer en el absolutismo, y por tanto en la imposibilidad de seguir pensando.

Desde posturas feministas diferentes (Weston, 1994; Hennessy, 1995; Fraser, 1995b, 1997; Kruks, 2001) se ha manifestado que precisamente la legitimación de las políticas feministas radica en la categoría "mujer" como una categoría que puede aglutinar experiencias compartidas de dominación basada en la identidad de género. Y por ello no está claro en Butler cómo sin esa categoría es posible accionar políticas feministas. Si bien estas posturas acuerdan que la categoría de género, y por tanto de mujer, es una categoría construida, no podrían aceptar la propuesta de Butler de que toda categoría, por tanto también la de sexo, es construida. Desde este feminismo más claramente universalista, la estabilidad y "fijación" de una categoría u otra (sexo o género) es necesaria para la lucha política feminista (Lloyd, 1999, 2005, 2007b; McNay, 1999; Mills 2000; Webster 2000; Smith 2001; Lovell 2003; Schwarzman 2002). Este debate ha estado claramente representado en las posturas enfrentadas de Judith $\mathrm{Bu}$ tler y Seyla Benhabib ${ }^{7}$.

${ }^{7}$ Para Benhabib (1995) de la teoría de la acción performativa de Butler no se sigue la posibilidad de un sujeto agente que sea capaz de luchar contra su propia subordinación. Esto lleva claramente, según Benhabib $(1995,20)$, a la crisis de identidad en el feminismo y aparta a éste de sus ideales tradicionales emancipatorios. El sujeto se reemplaza con un yo (self) fracturado y opaco (Benhabib, 1995, 16), y esto aleja a las mujeres de sus posibilidades como agentes, reduciéndolas a seres aún más frágiles. En el análisis de Fiona Webster (2000) de esta polémica se entiende que la distancia que separa a estas autoras está en que para Benhabib la agencia del sujeto radica principalmente en su reflexividad y en su autodeterminación. Para Butler no hay tal reflexividad intacta y autónoma en el sujeto dentro de un contexto cultural. Para Benhabib $(1992,8)$ los sujetos son situados (situated selfs) y tienen la capacidad de retar su situación para contribuir a la constitución de su propia identidad y a su propio lugar en el mundo. Desde esta visión la concepción performativa del sujeto que Butler da lleva a un determinismo contra el cual la agencia del sujeto simplemente ha desaparecido. En contrapartida lo que Butler cuestiona es precisamente que la misma estructura de lo político necesite de un sujeto estable. Desde las tesis nietzschianas de Butler (1995b, 46) las exigencias de un sujeto libre y autónomo, un "hacedor" detrás de los hechos, son una afirmación y demanda típicamente liberal, y que ha servido sólo para poder asignar responsabilidad y culpa al sujeto. Es decir, que sería simplemente una 
Para Butler la eficacia política de la performatividad de género es indudable. El poder performativo del signo "mujer" está precisamente en que actúa en aquello que nombra. Y por ello el carácter performativo del signo es su misma condición de su agencia (1995a, 150; 1995c, 134). La labor del feminismo, y de cualquier política emancipatoria, será encontrar las vías de desestabilizar los procesos a través de los cuáles somos construidos como sujetos, y al hacer eso abrir las posibilidades para el cambio y la transformación en nuestras identidades. Estas posibilidades siempre existen, pues, como Butler se ha encargado de repetir, la performatividad es un proceso dual: describe el mismo proceso de las normas al conformar al sujeto y a la vez alimenta la posibilidad de su propia resistencia.

La cuestión más débil en el entramado conceptual de Butler y su teoría performativa es precisamente explicar cómo incluso la resistencia y la transformación puedan llevar a una contestación real de las configuraciones de poder. Como la feminista Wendy Brown ha señalado (1995) la teoría performativa de Butler necesitará para ello introducir una estructura moral (entendida como un conjunto de aspiraciones normativas y de valores comunes, un objetivo común sobre "lo bueno", por ejemplo) si quiere llegar a la acción política eficaz; de otra forma carece de dirección política, lo cual es de vital importancia para la acción colectiva que el feminismo como práctica política necesita. Para Brown pues, queda claro que la mera resistencia no es estrategia suficiente para el programa político feminista, pues tiene el riesgo de no poder llegar a las aspiraciones colectivas de transformación que el feminismo necesita.

McNay $(1999,176)$ critica precisamente que el concepto de agencia en la noción de política performativa en Butler es demasiado abstracto y pobre de especificidad social. Butler necesitaría explicar cómo la teoría performativa trasciende la esfera privada para poder transformar los valores colectivos y las normas que constituyen nuestra identidad. Ha de dilucidar, además, cómo un sujeto construido en las redes de la sujeción puede llegar a actuar a través de prácticas de liberación. Este aspecto, esta carencia de una agencia autónoma para el sujeto, es el que lleva a feministas como Nancy Fraser a calificar a la filosofía de Butler como "profundamente anti-humanista" (1995a, 67).

En obras posteriores a sus dos libros más impactantes y seminales, de 1990 y 1993, Judith Butler ha ido respondiendo a estas críticas centradas en la poestructura ficticia establecida con propósitos morales (Butler, 1995c, 135). Para Butler el "hacedor" (doer) se constituye en y a través de los "hechos" (deed). La identidad es, por ello, una práctica de significación (Salih, 2002, 55-67). La teoría de la performatividad está encaminada justamente a captar el sentido en el que la significación y la acción se encuentran. Butler cree que el problema es que Benhabib no entiende su teoría de la performatividad; y por ello, expone en un ensayo titulado precisamente "For a Careful Reading" (1995c) que Benhabib reduce erróneamente la performatividad a la performance teatral, y ello colapsa y vuelve paródica, y en gran parte inútil a efectos políticos, a la performatividad. 
sibilidad de agencia del sujeto. Así, en Excitable Speech (1997a) Butler afirma que en los actos de habla hay siempre en cierta medida posibilidad de transgredir, de no reiterar la norma, de una apropiación no autorizada, y por tanto posibilidad de resignificación. Esta inestabilidad es lo que Butler llama "la excitabilidad del discurso". Ahí radica siempre la posibilidad del contra-discurso. Para Butler el poder performativo de una emisión no está sólo en su misma instantaneidad. Hay estructuras simbólicas y culturales de significación previas a la emisión que la permiten y la significan, y a la vez hay una posterior repetición y efecto de reiteración simbólica y cultural que genera un circuito somático de reconocimiento. (Butler 1997a, 3). Hay claramente una temporalidad abierta en el acto de habla que tiene como resultado una diseminación de los efectos más allá del control del sujeto hablante que puede dar lugar a la resignificación, a la supervivencia lingüística o a la muerte lingüística del hablante (Butler, 1997a, 28; McNay, 1999, 179).

Como conclusión de este primer apartado podemos decir que en la teoría performativa de género hay unos puntos de innegable avance para una teoría feminista:

1. Afirma la esencial inestabilidad de las significaciones históricas revelando el potencial de retar y transformar los sistemas patriarcales de significado.

2. Aleja la teoría feminista del esencialismo, empujándola a ir más allá de las polaridades del debate esencialista de la segunda ola del feminismo.

3. Ofrece una concepción discursiva de la identidad según la cual ésta nunca se construye de manera aislada de las normas dominantes y las formas ideológicas dadas previas al sujeto, ayudando con ello a explicar cómo a través de esos mecanismos discursivos se ha construido, entre otros sistemas normativos, el patriarcado, sistema hegemónico por excelencia.

Sin embargo, esta teoría también ofrece puntos a considerar para un ulterior análisis que pudiera explicar mejor cómo el marco conceptual de la teoría performativa de género puede servir para toda lucha y transformación por la igualdad. En concreto trataremos los tres puntos más débiles, y que pensamos pueden haberse resuelto en su última obra.

1. Se echa en falta en Butler una mayor presencia de las estructuras sociales y económicas y su relación con la esfera simbólica.

2. Falta una conexión más clara y evidente entre los resultados performativos individuales y los colectivos; cuestión vital para cualquier movimiento de liberación.

3. Necesita articular cómo desde la no-identidad podemos transformar las relaciones de poder entre grupos de actores sociales.

¿Pueden estos puntos débiles haberse superado en su última obra? 


\section{TEORÍA GENERAL: LA PERFORMATIVIDAD AMPLIADA}

Nótese que estos tres puntos débiles en la performatividad de género inciden en la misma cuestión: necesidad de abundar en la explicación de cómo la agencia del individuo está anclada en lo histórico y social, y por ende en lo colectivo; y vertebrar, a partir de ahí, una performatividad que sea eficiente en la transformación social y política.

Pensamos que esa explicación empieza a darla Butler en Undoing Gender (2004), donde en la misma introducción, llamada acertadamente "Acting in Concert" (en clara reminiscencia al pensamiento de Arendt), aclara la naturaleza social y comunitaria del acto performativo. Aunque en este libro Butler se centra principalmente en el activismo relacionado con la asignación de sexo, una de las conclusiones más importantes es la necesidad de crear normas y legislaciones que permitan una vida vivible, lejos de las restricciones que posibilitan sólo unas formas determinadas de vida (Butler 2004, 8).

La preocupación por las consecuencias de la falta de reconocimiento de una vida que no cuenta, se vuelve tema prioritario en Notes towards a Performative Theory of Assembly (2015b). En el primer capítulo Butler repasa resumidamente su teoría de la performatividad de género, tal y como la mantuvo hace 26 años. La conclusión más importante de esta teoría fue decir que el género sea performativo implica que el género es llevar a cabo algo, una promulgación, bien de las normas que lo constituyen y normalizan, bien de subversión de las mismas $(2015 b, 32)$. La aspiración política de Butler en su teoría de 1990 fue permitir que las vidas de las minorías de género y sexo pudieran ser más vivibles $(2015 b, 32)$. En este sentido Butler afirma en su último libro (2015b, 33) que su teoría de la performatividad en Gender Trouble ya contemplaba la precariedad, al entender el género y las minorías sexuales como vidas en condiciones invivibles y precarias. Aunque todo ser humano es vulnerable y está expuesto a precariedad, como afirma en 2015, hay una situación de distribución diferencial de esa precariedad en relación a las estructuras sociales y económicas $^{8}$. Estas se traducen en normas que condicionan nuestras vidas y las hacen vivibles o invivibles. El necesario reconocimiento que toda norma implica

\footnotetext{
${ }^{8}$ Es pertinente en este punto distinguir, como lo hace Butler en Frames of War (2009), entre "precariousness" y "precarity". Este último término hace referencia a la distribución desigual y políticamente inducida de la precariedad. La precariedad (precariousness) sería, en contrapartida, la condición metafísica de todo ser humano. En castellano no podemos traducir esta diferencia sin crear un neologismo (precaridad). En el libro de Notes towards a Performative Theory of Assembly (2015b) la autora utiliza genaralmente le término de "precarity", pues precisamente nos habla de la precariedad inducida. Hemos decidido utilizar el término aceptado en castellano de "precariedad" y expresar o explicar la diferencia, cuando la haya, en el contexto mismo de la frase.
} 
no sólo se entiende ahora en relación a sexos y géneros minorizados, sino a cualquier aspecto que regule la "aparición" de los sujetos. Materializar las normas en nuestros cuerpos nos hace ganar estatus y reconocimiento como sujetos, y ello, a su vez, legitima unas determinadas normas de reconocimiento, y constriñe finalmente el campo de lo "reconocible”: "¿Por qué es ese campo regulado de tal forma que sólo ciertas clases de ser pueden aparecer como sujetos reconocibles y otros no pueden?"9 $(2015 b, 35)$. ¿Quién, en definitiva, tiene derecho a aparecer? Esta pregunta se resuelve en una de más calado y que Butler viene arrastrando desde el inicio de su reflexión filosófica: ¿Qué humanos cuentan como humanos? Este interrogante lleva a Butler a plantear una contradicción, y es que son las normas, y su performatividad, las que nos dicen que hay humanos que no cuentan como humanos. Esta contradicción permite mantener una desigualdad aceptada. La propuesta de Butler a lo largo de sus obras ha sido precisamente interpretar la performatividad en las normas (las de género, pero también otras) como posibilidad de reformular constantemente el campo de lo humano, sin tomar como cerrado el proceso normativo y social que lleva a estructurar las vidas humanas. Son los cuerpos los que apelan a las normas de reconocimiento para desmantelar formas opresivas de poder, para meterse por las rendijas de los sistemas normativos e invalidarlos, o al menos cuestionarlos. Es en la aparición y contestación de los cuerpos que las normas se rompen y se abren en nuevas formas $(2015 b, 37)$.

La performatividad de lo humano, en sus múltiples facetas, es entendida por Butler en términos hegelianos. En este sentido sea lo que sea lo que denominemos vida humana ha de estar en relación con una tensión continuada entre dos términos, humano e inhumano. Es más, Butler afirma que precisamente es esa negociación continua lo que podemos denominar "humano" (2015b, 43). Por ello en la negociación de lo humano no sólo entran aspectos mantenidos por otros y que podamos pensar como no-humanos, sino las infraestructuras que organizan y sostienen la vida, incluido el medio ambiente. La misma capacidad de acción que me convierte en sujeto depende de otros y de las condiciones de vida que se dan en el proceso de creación de vida humana en colectividad. La dimensión ética no sólo se da así en la mera pregunta de cómo he de vivir mi vida, sino en la cuestión política de cómo hemos de vivir conjuntamente. La cuestión de lo que hace que una vida sea vivible es así previa a la cuestión de qué clase de vida puedo vivir. Y esto conlleva, irremediablemente, tomar en serio las condiciones biopolíticas de las normativas que permiten desarrollar la vida (no sólo la humana).

${ }^{9}$ Traducción propia de: "Why is that field regulated in such a way that only certain kinds of being can appear as recognizable subjects and others cannot?" 
Las condiciones de interdependencia para la vida son así las verdaderas posibilitadoras de la vida misma, y esas condiciones, Butler afirma en su último libro, están amenazadas por el régimen político y económico actual. Esta idea rompe definitivamente con la presuposición arendtiana expuesta en La condición humana (1958) de separar lo privado de lo público, en el sentido de relacionar la esfera pública con lo político a través del acto de habla, y la esfera privada con el cuerpo. Para Butler, y tomando en cuenta las críticas feministas a esta separación, sobre todo las de Zerilli (1995) y Coks (1995), es el cuerpo y la esfera privada lo que permite hablar del ciudadano varón y la esfera pública. Butler toma cuenta de la importancia del cuerpo para Arendt, quien en Sobre la revolución (1963a) reconoció que hay movimientos políticos surgidos desde la necesidad del cuerpo. Ahora bien, para Arendt, aun tomando en consideración esto, estos movimientos están motivados por la necesidad y no por la libertad. Ello deja, según la visión política de los cuerpos de Butler, no sólo separados el cuerpo de la mente/habla, sino la dimensión política de la económica: "Si el cuerpo permanece en el nivel de la necesidad, entonces parecería que ninguna explicación política de la libertad puede ser encarnada" 10 (2015b, 47). La libertad ha de reconciliarse con la necesidad, y es ahí donde ampliamos la noción de lo político, aliamos lo político con lo económico, conectamos el cuerpo con la mente, y hacemos dependiente la precariedad con la performatividad de la vida. El mismo "derecho a tener derechos" que Arendt mantiene sirve para poder hacer esta reconciliación, ya que permite entender un elemento corporal en la resistencia política del simple cuerpo que clama ese derecho. Son precisamente aquellos humanos que son considerados "inelegibles" (los que no cuentan) los que necesitan partir de una dimensión de mera aparición de su cuerpo para poder resistirse y transformar las normas, para criticar las formas diferenciales de poder, para exigir el derecho a tener derechos. Con la mera aparición del cuerpo ya se reclama igualdad, y ello lo convierte en un acto político. La mera presencia y congregación de cuerpos ha de ser entendida como resistencia a un poder que dice que mi sola presencia no es política y no me habilita como sujeto político. Creemos que aquí radica la esencia de la teoría de la performatividad de Butler, que ahora hemos llamado "performatividad ampliada" o "performatividad general".

La precariedad (precarity) es la situación que congrega las condiciones de vida no vivibles por cuestión de género, sexualidad, sexo, etnia, raza, religión, estado, nación, situación económica...Esta es la idea que permite una ampliación de la teoría de la performatividad de género a toda situación de vida precaria. El

${ }^{10}$ Traducción propia de: "If the body remains at the level of necessity, then it would appear that no political account of freedom can be an embodied one". 
punto de arranque no es la identidad, sino las condiciones sociales y económicas que marcan las formas diferenciales del poder. La idea la resume Butler cuando nos dice que no sólo es que alguien tiene que vivir para actuar, sino que ese alguien tiene que actuar políticamente para asegurar las condiciones de su existencia $(2015 b, 58)$. Y, por ello, la promulgación en un acto performativo es una manera de repensar la ontología $(2015 b, 61)$. Butler toma la oportunidad de aclarar con esta explicación la afirmación de que el "género es performativo", pues, como ya hemos indicado fue objeto de interpretaciones contradictorias y, a la postre, equivocadas. Algo anda mal, reconoce Butler, cuando su afirmación se pudo entender de dos formas tan dispares, voluntarista o determinista. La performatividad es dual, pues describe tanto el proceso de actuar sobre el género como el de las posibilidades y condiciones de actuar; y por ello, ni podemos rehacerlo a voluntad ni estamos negadas en nuestra capacidad de acción por el mismo (Butler, 2014). El cuerpo no se resume en una identidad, sino que es más bien un "conjunto viviente de relaciones" $(2015 b, 65)$, está atado a condiciones de infraestructura y medioambientales, así como a otros cuerpos y a poderes que le reconocen o le niegan. Actuar siempre es posible, bien sea con esas condiciones a favor o en contra. Precisamente es la acción con esas condiciones en contra la paradoja que Butler cree interesante pensar. Su teoría de la acción performativa plural (y ampliada) pretende entender cómo es posible actuar en condiciones de precariedad, y por tanto, alejar la acción performativa tanto de la mera voluntariedad como de la absoluta imposibilidad.

Butler reconoce que estamos en en un escenario lleno de constreñimientos sociales y culturales. El sujeto se encuentra en una socialidad que no remite a un único autor (Butler, 2004, 1). La actividad incesante de performar mi identidad está siempre en relación con otros, incluso si estos son sólo imaginarios. Así, la práctica performativa es esencialmente plural y congrega una pluralidad de cuerpos; lo que Butler llama en su obra de 2015 "la asamblea pública de cuerpos". Y es este punto, ya presente en su obra de 1990, que ahora acentúa, desarrolla y profundiza, el que nos permite hablar de una teoría performativa ampliada.

Esta ampliación de la teoría performativa plural a la asamblea, tiene dos fuentes principales como base teórica:

1. El concepto de Arendt de lo político como "espacio de aparición”. Es decir, que Butler toma de Arendt la base de lo político en lo público. Lo político se da en el espacio "entre la gente" (Butler, 2015b, 73), o "Zwischen-Raum" en denominación de Arendt. Ese espacio no se da de forma previa al encuentro, sino que se conforma precisamente en la misma aparición del acto público de congregación o asamblea. Lo que Butler (Bu- 
tler \& Athanasiou, 2013, 179-180) añade a esta concepción arendtiana es justamente la existencia corporal en el espacio público como elemento esencial de la política democrática, sacando así al cuerpo de la esfera privada. El lema de Arendt de "el derecho a tener derechos" se reinterpreta para ampliarlo a aquellos que necesitan unirse, aliarse, para ser siquiera aptos para tener derechos. La libertad y la igualdad para los/las precarios/as son concebibles únicamente de forma performativa, pues se muestran a su alcance solamente a través de las congregaciones de cuerpos, como ya se encargó de señalar Butler en Frames of War (2009, vii).

2. La teoría performativa de género, la cual nos permite entender ahora que también las asambleas, como congregaciones de cuerpos, son performativas, puesto que son esencialmente actos corporales que muestran que cualquier existencia corporal humana es una existencia de "ser-con" (being-with). Por ello, la performatividad de un cuerpo es, deviene y hace, con otros cuerpos; y a la vez depende de condiciones impuestas, no sólo de los otros cuerpos, sino de recursos materiales, psíquicos, culturales, económicos, jurídicos... Y de ahí que Butler afirme que una asamblea de cuerpos tiene una disposición performativa que conlleva tanto elementos pasivos como activos, ya que comprende "tanto los procesos de actuación como las condiciones y posibilidades de actuación"11 $(2015 \mathrm{~b}, 63)$.

Con esta base teórica nos preguntamos: ¿qué hace política a una asamblea de cuerpos?

Para contestar a esto necesitamos el concepto de precariedad. Butler cree que la radicalización del régimen neoliberal en las últimas dos décadas le ha convertido en un régimen que hace a cada individuo responsable de la producción y reproducción, como fuerza laboral, de su devenir social y económico. La racionalidad neoliberal "demanda auto-suficiencia" (Butler, 2015b, 14, 144). La responsabilidad es reapropiada por el neoliberalismo como moralidad que se centra en la autosuficiencia económica, entendida desde unas condiciones estructuralmente determinadas exclusivamente en lo económico (Butler, 2015b, 25). Esta exigencia de autosuficiencia conlleva que el individuo tenga permanentemente que encararse con demandas que nunca pueden ser satisfechas del todo. Aquellos y aquellas que pueden entrar en este juego son sujetos cuyas vidas "son vivibles", pero Butler se interesa por aquellos y aquellas cuya condición, por razones subjetivas y objetivas, no pueden "jugar este juego". Ello les convierte en "desechables" (disposable), en seres precarios. La precariedad

${ }^{11}$ Traducción propia de: "both the processes of being acted on and the conditions and possibilities for acting". 
inducida (precarity) se define así como la condición política a la que se ve abocada cierta parte de la población que sufre al no poder entrar en las redes sociales y económicas que el sistema decide. Ello les hace estar expuestos a "lesión, violencia y muerte" $(2015 b, 33)^{12}$. Las congregaciones o asambleas de cuerpos se convierten así en la respuesta, ahora ya no sólo fenomenológica, sino política, a esta condición de vulnerabilidad y desechabilidad. La contra-práctica de los cuerpos, congregados en los espacios públicos, son prácticas tanto de solidaridad como de igualdad $(2015 b, 66)$, que demandan su "derecho a aparecer" en un régimen neoliberal que les está condenando a la invisibilidad pública.

La manera de responder y mostrar resistencia a los procesos de precarización actuales será desactivando esa idea de responsabilidad individual de la situación que me convierte en precario/ $\mathrm{a}^{13}$. Y por ello la asamblea es la forma de actuar que desestabiliza esa idea neoliberal de responsabilidad al decir, aun sin hablar, con la mera congregación en el espacio público, "no somos desechables" (Butler, 2015b, 25). Frente a este individualismo neo-liberal Butler ya afirmó en Frames of War (2009) que la precariedad no es una "verdad existencial". To-

${ }^{12}$ Butler, siguiendo el concepto de biopolítica de Michel Foucault y el de necropolítica de Achille Mbembe, anota cómo, no sólo hay políticas que explícitamente buscan la muerte de ciertas poblaciones, sino que también hay políticas que produciendo condiciones de negligencia y desamparo dejan que la gente muera (2015b, 11-12)

${ }^{13}$ En el momento de escribir este texto (noviembre 2016) ha saltado la noticia en todos los medios de comunicación en España del caso de una anciana muerta por asfixia en su casa debido a un incendio por unas velas. La situación fue provocada por el corte de suministro eléctrico dos meses antes por impago. La autoridad local acusa a la compañía de suministro eléctrico. Esta, a su vez, acusa a la autoridad local por no facilitar un protocolo de actuación para estos casos. Las acusaciones cruzadas muestran claramente los signos de nuestros tiempos y a los que Butler refiere con el término "condiciones de negligencia que hacen que la gente muera". En el caso específico aquí relatado la muerte de una persona en condición de precariedad extrema es traducida en acusaciones de mala gestión, alejándose así de la responsabilidad de una vida, y finalmente de posibles cuestiones morales que la responsabilidad conlleva. El proceso de vaciedad de sentido continua así en los medios al contrastar los conceptos de "vida humana" con "protocolo de actuación". La posible propuesta futura de un "protocolo de actuación" consensuado por autoridades y compañías eléctricas de alguna manera "normalizará" la precariedad de las y los que no pueden pagar la factura eléctrica. En el caso que Butler trata en su libro, el de la falta de seguro médico de millones de precarios en USA, tilda de "joyous relation to cruelty" (jubilosa relación con la crueldad) a la falta de responsabilidad social común que hay en quienes miden y calculan, desde la racionalidad económica, para objetar la asistencia pública sanitaria (2015b, 11-14). La anciana que ha fallecido no fue auto-suficiente, no pudo hacer frente a "sus" facturas; y ello la volvió dispensable, desechable. La democracia social se diluye así entre la responsabilidad individual del precario/a y la gestión empresarial de los servicios privatizados del suministro eléctrico. La responsabilidad se redefine así, como Butler $(2015 b, 15)$ afirma, "como la demanda a convertirse en un/a emprendedor/a de sí mismo/a bajo condiciones que hacen imposible esa dudosa vocación". 
dos estamos expuestos, todos somos vulnerables, aunque en niveles diferentes. Ello corrobora la idea de la dependencia humana de infraestructuras de apoyo y mantenimiento de la vida. La estructura que organiza la vida en las sociedades está íntimamente ligada al sentido de la vida individual (Butler, 2015b, 21), y por ello en cada "fallo" individual que lleva a condiciones de precariedad hay organizado un "fallo" de esas infraestructuras. En el mundo neoliberal actual estos "fallos" son parte de las estructuras de las instituciones políticas y socioeconómicas. Esta reflexión aviva la idea de un ethos de la solidaridad y nos aparta de la forma "enloquecedora" de la responsabilidad individual en la precariedad (Butler, 2015b, 21). Como quiera que sea nuestra vida, buena o mala, más o menos precaria, está siempre vinculada a la vida social, implicada en una infraestructura del mundo que excede al individuo (Butler, 2015b, 23). La vulnerabilidad y la posibilidad de precariedad son constantes y generalizadas; son condiciones mismas de la vida humana en interdependencia; es más, en radical dependencia de unos/as con otros/as y con el mundo y planeta (Butler, 2015b, 152). Para Butler (2015b, 123-153) ello tiene claras consecuencias políticas: puede convertirse en la base, y a la vez en el objetivo, de políticas de coalición que den forma a la acción conjunta de los cuerpos que se movilizan en asamblea ${ }^{14}$. La vulnerabilidad y la agencia van juntas, nos dice Butler (2015b, 139), convirtiendo esta idea en la base de su política performativa (tanto en la de 1990 como en la de 2015) ${ }^{15}$. La vulnerabilidad, individual, pero también colectiva, ha de activar la agencia y es la base de políticas democráticas.

En las circunstancias de devastación de lo público y de exclusiva responsabilidad del individuo las condiciones para actuar juntos son constantemente socavadas. Por ello, la emergencia de asambleas, de resistencias en grupo, de solidaridad social, son a la vez tristes y alegres, nos dice Butler (2015b, 23). Tristes porque se dan en situación de precariedad, alegres porque significan resistencia y solidaridad. De alguna manera esto puede darle un acento de fragilidad a la idea de cuerpo precario; aunque es esa precariedad de los cuerpos la que activa la situación política de resistencia de los mismos, indicando así un camino para la acción. El carácter político de estas asambleas de cuerpos proviene precisamente de ese carácter congregacional que responde y resiste a la producción de precariedad del actual régimen neoliberal. Estas asambleas ne-

${ }^{14}$ Es importante aclarar que para Butler una asamblea no necesariamente es una congregación de cuerpos en un espacio físico, como la calle o una plaza; puede ser también una congregación virtual de cuerpos.

${ }^{15}$ De esta manera la categoría butleriana de "mujer", que tantos debates originó, como hemos visto más arriba, se resuelve ahora en relación al tándem vulnerabilidad-agencia: "mujeres" es el nombre de un grupo de personas que son más vulnerables, pues están más expuestas a la discriminación, acoso y violencia (Butler 2015b, 142). 
cesitan un constante esfuerzo por trascender la particularidad de su identidad, yendo más allá de las políticas identitarias de otras épocas. De hecho, este es un punto que no ha cambiado en los veintiseis años entre las dos obras que aquí analizamos. Como la misma Butler afirma $(2015 \mathrm{~b}, 27)^{16}$ :

Un punto político, probablemente, ha permanecido muy parecido incluso cuando mi propio enfoque se ha desplazado; y es que la política identitaria falla al no porporcionar una concepción más amplia de lo que significa, políticamente, vivir juntos, a través de las diferencias, a veces en modos de proximidad no elegidos, especialmente cuando convivir, por difícil que sea, sigue siendo un imperativo ético y político.

Para accionar la carga política de una comunidad de cuerpos ésta ha de trascenderse a sí misma, creando vínculos y solidaridad con otras comunidades, con otras asambleas de cuerpos, con las cuáles se comparte la misma condición social de precariedad. Una asamblea de cuerpos con una demanda concreta va más allá de la misma al ser una demanda por la justicia, por el reconocimiento, por el derecho a aparecer, por una vida vivible. Cada asamblea está así en relación con otras asambleas, con las cuáles comparte, al menos, estas últimas demandas citadas. Podemos decir que cada asamblea es una asamblea de asambleas, convirtiéndose en el sujeto político de la política radical. Sólo en esa unión las asambleas pueden ser capaces de resolver las cuestiones de precariedad actual.

Los movimientos no son permanentes y estables, son transitorios, y por ello están conectados a la función crítica que tienen (Butler, 2015b, 20). Así, no es posible acusar, como constantemente se hace, a una asamblea de que no ha permanecido en el tiempo, de que no ha durado, y que por tanto ha sido inútil. Las asambleas pueden aparecer en cualquier momento, y ahí radica su fuerza crítica. Al mismo tiempo las acusaciones repetidas a estas asambleas de que parecen no tener demandas claras y consistentes, que no tienen una ideología clara o un programa articulado, y que por ello no pueden ser interpretadas como expresiones políticas, es un error. Butler propone un entendimiento amplio de lo que significa "expresión política". En su propuesta la mera congregación de cuerpos en un espacio público es una expresión política que supera la visión clásica de la acción política en la forma tradicional verbal, llevada a cabo en parlamentos o en agrupamientos en los que se vocalizan las propuestas. Las condiciones de aparición no sólo son las manifestaciones vocales, sino también las

${ }^{16}$ Traducción propia de: "One political point probably has remained pretty much the same even as my own focus has shifted, and that is that identity politics fails to furnish a broader conception of what it means, politically, to live together, across differences, sometimes in modes of unchosen proximity, especially when living together, however difficult it may be, remains an ethical and political imperative". 
condiciones de puesta en escena, los medios tecnológicos de captar las imágenes de la congregación (video-cámaras, móviles, cámaras de vigilancia...), así como los sonidos, los signos gráficos que se utilizan. Es decir, toda la actividad de auto-constitución en el espacio público (Butler, 2015b, 19-20).

Precisamente al ser actos corporales desde la precariedad son ya expresiones políticas. Con ello Butler supera los límites de la filosofía de Arendt para entender qué es política, y enlaza lo político inextricablemente con los cuerpos, sus necesidades y sus deseos. Así, las luchas, enlazadas con la esfera del trabajo, realizadas por trabajadores/as empobrecidos/as, trabajadores/as migrantes, trabajadores/as sin derechos, parados/as, que ven mermadas sus condiciones de vida por un sistema neoliberal que hace del trabajo fuente de precariedad, son luchas que buscan alcanzar las necesidades básicas de supervivencia. Y esta es probablemente la lucha política de nuestro tiempo.

Este es uno de los aspectos más notorios de la obra de 2015, en el sentido de que la mirada de Butler a la performatividad excede claramente la palabra y la representación. Al ampliar lo político a la mera aparición de la materialidad de los cuerpos congregados amplía su teoría de la performatividad más allá de la fenomenología política feminista que elaboró en Gender Trouble. La materialidad de las asambleas es política en cuanto interpela, con su derecho a persistir, las condiciones con las que el aparato del estado regula el espacio público y el derecho de aparición. Esta nueva ampliación de la política performativa de Butler implica una visión de la nueva ética global articulada en la "necesidad que tenemos de los otros" (2015b, 218). Con ello, y tomando como referencia la ética de la responsabilidad de Emmanuel Levinas ${ }^{17}$, elabora su propia propuesta ética.

Según ésta, y yendo más allá de Levinas, en el mundo global el sujeto ético no es reducible a su pertenencia nacional o afiliación comunitaria: "la vida del otro/a, la vida que no es la propia, es también nuestra vida..." ${ }^{18}(2015 b, 108)$. Esta forma de responsabilidad ética no es, sin duda, fácil de llevar a cabo cuando alguien se siente precario/a, y que su vida no es vivible. Y ello, esa precariedad, es lo que suscita ambas: la necesidad y la dificultad de la ética (Butler, 2015b, 109). La relación ética precede al individuo, como Levinas ya afirmó, y a la vez que lo conforma como "yo" ético, lo desposee como yo: "I am undone as a bounded

${ }^{17}$ Butler se ha servido a lo largo de sus obras del pensamiento judío de autores como Arendt o Levinas. Butler recoge la parte de la tradición judía moderna (Jewishness) que escapa del esencialismo (Judaic), y que permite, a través de una "ética de diáspora" y del cosmopolitismo, confrontar la naturaleza etnocéntrica y nacionalista de ciertos discursos judíos actuales (Judaic). Es en 2012, con su libro Parting ways, que desarrolla estas ideas críticas, profundizando para ello en el concepto mismo de convivencia.

${ }^{18}$ Traducción propia de: "the life of the other, the life that is not our own, is also our life..." 
being" $(2015 b, 110)$. Esto sitúa a la ética que desarrolla Butler en el terreno que Arendt emprendió en Eichmann en Jerusalén (1963b), y que es el de la necesaria convivencia con los que existen. Este es el límite de la libertad para Arendt y también para Butler, y lo que convierte a la libertad en una acción plural, política y social. La libertad individual no puede darse si no es aceptando esta condición de convivencia, que a su vez garantiza la pluralidad, la posibilidad de nuestro estatus como personas y la política misma. El carácter no-elegido de esta condición ha de ser activamente preservado por las instituciones políticas, y para Butler esta conclusión de Arendt nos lleva a poder hablar de normas políticas concretas que se pueden derivar de ella (Butler, 2015b, 113). Políticas que han de preservar con la misma obligatoriedad la pluralidad y la igualdad, y en definitiva la universalidad. En este sentido de la ética de la convivencia es que Butler declara en una sugerente frase "Todos somos, en este sentido, los no elegidos, aunque somos no-elegidos juntos"19 $(2015 \mathrm{~b}, 116)$. La dimensión precaria de la vida, de la que no escapa nadie, es el fundamento de esa obligación ética de la convivencia no-elegida. Butler se esfuerza en su último libro por ofrecer una visión más clara de cómo esa interdependencia, en una vida vivible y en la precariedad, puede llevarse a cabo de manera sostenible en convivencia, y además en términos igualitarios $(2015 b, 120)$. Las obligaciones éticas que emergen de la propuesta que Butler (2015b, 121-122) hace en relación a su teoría de la performatividad ampliada o general son, por tanto, tres:

1. Hay que honrar las obligaciones que preservan la vida de aquellos con quienes habitamos el planeta. No importa si no los conocemos, o no los elegimos.

2. Hay que luchar por unos ideales que estén en consonancia con la situación material creada por las condiciones sociales de la política y la economía. Por ello la deliberación y el acuerdo por una vida buena no son siempre suficientes.

3. Hay que luchar por encontrar un entendimiento global sobre formas de vida económica y políticamente vivibles, alejadas de la precariedad (precarity), y políticamente en igualdad.

Con una ética de este cariz Butler rechaza el individualismo burgués, aclarando que "la fuerza de lo que todavía podemos llamar ética" $(2015 \mathrm{~b}, 122)$ radica en que el nosotros, vosotros, el aquí, allí, la proximidad o distancia, entran en conexión. No tenemos elección: "Vivimos juntos porque no tenemos elección" (Butler, 2015b, 122).

${ }^{19}$ Traducción propia de: "We are all, in this sense, the unchose, but we are nevertheless unchosen together". 


\section{CONCLUSIONES}

En el presente escrito hemos elaborado un análisis de la propuesta reciente de Judith Butler sobre la performatividad de la agencia política, lo que hemos llamado "performatividad ampliada o general". Para ello hemos evaluado principalmente las ideas defendidas en su último libro, Notes towards a Performative Theory of Assembly (2015b). La tesis principal del libro parte de ideas defendidas en escritos previos ${ }^{20}$, aun cuando hayan sido mal interpretadas o no suficientemente explicadas. Entre estas ideas encontramos la crítica a la falsa división entre pensamiento y acción, que se formaliza en la tesis butleriana de que el pensamiento está corporeizado, materializado. Y que lo está en normas de vida vivibles, y ahí en las normas de lo vivible, de lo que cuenta como una vida y lo que no, y en la práctica asociada de la responsabilidad crítica según el principio de convivencia desarrollado por Butler, encontramos la normatividad necesaria para una teoría ética y política.

Si en Gender Trouble Butler hablaba de actos que podían tener efectos subversivos en la performación de las normas de género, en el último libro lo que le interesa a Butler es la posibilidad de las alianzas entre minorías o poblaciones que son estimadas como "desechables". Es decir, como ella misma explica (2015b, 27), cómo la precariedad opera como elemento de alianzas entre gentes que en principio puede que no tengan mucho en común, o incluso que se muestren antagónicos. Pese a esta ampliación de la teoría de la performatividad en Butler el concepto de agencia, ahora explícitamente vinculado a vulnerabilidad y precariedad ${ }^{21}$, sigue entendiéndose como práctica performativa de subversión.

Basándose en estas ideas Butler amplía, en su último libro, la teoría de la performatividad a acciones que no son puramente actos de habla, ni sólo actos de hacer o deshacer el género. La amplía, precisamente a la asamblea y a las acciones que congregan cuerpos que expresan resistencia. Estas formas corporeizadas de congregarse implicarán una nueva manera de entender el espacio público y la política. Y con ello, pensamos, da respuesta a los tres puntos débiles que habíamos señalado en el segundo apartado de este escrito. Es decir, 1) Amplía la teoría de la performatividad a un contexto amplio de relaciones socio-económicas. De hecho, al comprender una teoría de la performati-

${ }^{20}$ Aunque la mayoría de los seis capítulos fueron desarrollados previamente como conferencias en Bryn Mawr College en 2011, como parte de las Mary Flexner Lectures, y algunos de ellos (cuatro) fueron publicados previamente al libro en forma de artículos para revistas o volúmenes editados, es la primera vez que se publican conjuntamente.

${ }^{21}$ Hay que recordar, sin embargo, que Butler ya habló de precariedad en libros tan anteriores como Undoing Gender (2004) y Giving an account of oneself (2005). 
vidad vinculada a la precariedad, Butler intenta dar respuesta a las vidas no vivibles que genera el sistema económico social del neoliberalismo. 2) Muestra claramente cómo las acciones y resultados performativos individuales y los colectivos están aunados. Creando, de hecho, una teoría ética de la convivencia que comprende al sujeto en dependencia con otros (tanto sujetos como sistemas). 3) Propone una teoría política performativa que permite incidir en cambios en las relaciones de poder, conllevando posibilidades de transformación colectiva (e individual) tanto en el espacio público como en el privado.

Con las nuevas explicaciones de la teoría de la performatividad pensamos que Butler puede superar finalmente las acusaciones repetidas (y contrapuestas) a su teoría de la performatividad de género en el sentido de que proponían un sujeto bien totalmente determinado y sin posibilidad de agencia o, bien que esa agencia parecía basada en un simple voluntarismo. Como conclusión podemos decir que en la ampliación e interconexión de las luchas del contexto actual la performatividad se resuelve operando en los intersticios que producen ambos, lo querido y lo no querido (Ahmed \& Butler, 2016, 483). La performatividad es un tránsito constante entre la determinación de las normas (ahora, y de forma clara, no sólo las de género y sexo) y la voluntad de transformación. La vulnerabilidad y precariedad de los cuerpos son el motor que nos mueve a encontrar/producir esos intersticios para crear juntos las condiciones de una vida vivible. Las condiciones críticas de nuestro tiempo nos lo exigen con urgencia.

\section{BIBLIOGRAFÍA}

Ahmed, Sara \& Butler, Judith (2016) “Interview with Judith Butler”, Sexualities, 9(4), pp. 482-492.

Arendt, Hannah (1958) La condición humana, edición de 1993, Barcelona, Paidós (Trad. Ramón Gil).

Arendt, Hannah (1963a) Sobre la revolución, edición de 2004, Madrid, Alianza (Trad. Pedro Bravo).

Arendt, Hannah (1963b) Eichmann en Jerusalén, edición de 2003, Barcelona, Lumen (Trad. de Carlos Ribalta).

Austin, John (1962) How to do things with words, Oxford University Press.

Bell, Vicky, ed. (1999a) Performativity and Belonging, London, Sage Publications.

Bell, Vicky (1999b) "On speech, race and melancholia. An interview with Judith Butler", Theory, Culture and Society, 16(2), pp. 163-174. 
Benhabib, Seyla (1992) Situating the self: Gender, community and postmodernism in contemporary ethics, Oxford, Blackwell.

Benhabib, Seyla (1995) "Feminism and Postmodernism”, en Benhabib, Seyla; Butler, Judith; Cornell, Drucilla \& Fraser, Nancy, 1995, pp. 17-34.

Benhabib, Seyla; Butler, Judith; Cornell, Drucilla \& Fraser, Nancy (1995) Feminist Contentions: a Philosophical exchange, New York, Routledge.

Bigwood, Carol (1998) "Renaturalizing the Body (with the Help of Merleau-Ponty)", en Welton, Donn, ed., 1998, pp. 99-114.

Birkenstein, Cathy (2010) "Reconsiderations: We Got the Wrong Gal: Rethinking the 'Bad? Academic Writing of Judith Butler". College English, 72(3), pp. 269-283.

Bordo, Susan (1993) Unbearable Weight: Feminism, Western Culture, and the Body, The Regents of the University of California.

Boynton, Robert (1999) “Who Needs Philosophy?", The New York Times (21 November 1999). <http://www.nytimes.com/1999/11/21/magazine/who-needs-philosophy.html>

Brown, Wendy (1995) States of injury: Power and freedom in late modernity, Princeton, University Press.

Burgos Diaz, Elvira (2008) Qué cuenta como una vida. La pregunta por la libertad en Judith Butler, Madrid, Antonio Machado Libros.

Burgos Diaz, Elvira (2012) “Deconstrucción y Subversión”, en Soley-Beltrán, Patricia \& Sbasay, Leticia, eds., 2012b, pp. 101-133.

Butler, Judith (1990) Gender Trouble: Feminism and the subversion of identity, New York, Routledge.

Butler, Judith (1991a) “Imitation and gender insubordination”, en Fuss, Diana, ed., 1991, pp. 13-31.

Butler, Judith (1991b) "Contingent foundations: Feminism and the question of postmodernism”, Praxis International, 11 (2), pp. 150-165.

Butler, Judith (1991c) "Response to Teri Shearer's review of Gender Trouble: Feminism and the Subversion of Identity", The Journal of Social Epistemology, 5(4), pp. 345-8.

Butler, Judith (1993) Bodies that matter: On the discursive limits of sex, New York, Routledge.

Butler, Judith (1995a) “Burning acts: Injurious speech”, en Haverkamp, Anselm, ed., 1995, pp. 149-180.

Butler, Judith (1995b) "Contingent foundations”, en Benhabib, Seyla; Butler, Judith; Cornell, Drucilla \& Fraser, Nancy, 1995, pp. 35-48.

Butler, Judith (1995c) "For a careful reading”, en Benhabib, Seyla; Butler, Judith; Cornell, Drucilla \& Fraser, Nancy, 1995, pp. 127-144. 
Butler, Judith (1997a) Excitable Speech: A politics of the Performative, New York, Routledge.

Butler, Judith (1997b) The Psychic Life of Power, Stanford University Press.

Butler, Judith (1998) “How Bodies come to Matter: An Interview with Judith Butler”, Signs. Journal of Women in Culture and Society, 23(2), pp. 275-286 (Entrevista con Irene Costera Meijer y Baukje Prins).

Butler, Judith (1999a) Gender Trouble: Feminism and the subversion of identity (segunda edición), New York, Routledge.

Butler, Judith (1999b) “A 'Bad Writer' Bites Back”, The New York Times (20 March 1999). <http:/query.nytimes.com/gst/fullpage.html?res=950CE5D61531F933A15750C 0A96F958260>

Butler, Judith (2000) "Ethical Ambivalence”, en Garber, Marjorie; Hanssen, Beatrice; Walkowitz, Rebecca L., eds., 2000, pp.15-28.

Butler, Judith (2003) "Values of Difficulty”, en Culler, Jonathan \& Lamb, Kevin, eds., pp. 199-215.

Butler, Judith (2004) Undoing Gender, London, Routledge.

Butler, Judith (2005) Giving an Account of Oneself, New York, Fordham University Press.

Butler, Judith (2009) Frames of War. When Is Life Grievable?, London \& New York, Verso.

Butler, Judith (2012) Parting Ways: Jewishness and the Critique of Zionism, Columbia University Press.

Butler, Judith (2014) "Rethinking Vulnerability and Resistance", ponencia presentada en Universidad Alacalá de Henares.

Butler, Judith (2015a) Senses of the Subject, New York, Fordham University Press.

Butler, Judith (2015b) Notes towards a Performative Theory of Assembly, Harvard University Press.

Butler, Judith \& Athanasiou, Athena (2013) Dispossession: the Performative in the Political (Conversations Butler \& Athanasiou), Cambridge, Polity Press.

Butler, Judith \& Connolly, William (2000) "Politics, Power and Ethics: A Discussion between Judith Butler and William Connolly", Theory \& Event, 4(2). http://muse.jhu.edu/ journals/theory_and_event/v004/4.2butler.html

Carver, Terrel \& Chambers, Samuel A. eds. (2008) Judith Butler's Precarious Politics: Critical Encounters, New York, Routledge. Milton Park, Abingdon, Oxon.

Chambers, Samuel A. \& Carver, Terrell (2008) Judith Butler and Political Theory: Troubling Politics, London, Routledge.

Cocks, Joan (1995) “On Nationalism”, en Feminist Interpretations of Hannah Arendt, Honig, Bonnie, ed., Penn State University Press, pp. 221-246.

Culbertson, Carolyn (2013) "The ethics of relationality: Judith Butler and social critique", Contemporary Philosophical Review, 46, pp. 449-463. 
Culler, Jonathan \& Lamb, Kevin, eds. (2003) Just Being Difficult. Academic Writting in Public Arena, Standfors University Press.

Derrida, Jacques (1972) “signature événement contexte”, en Derrida, Jacques, (1994) Márgenes de la Filosofía, Madrid, Cátedra, pp. 347-372, (Traducción de Carmen González Marín.

Fraser, Nancy (1995a), "False Antithesis: a response to Seyla Benhabib and Judith Butler", en Benhabib, Seyla; Butler, Judith; Cornell, Drucilla \& Fraser, Nancy, 1995, pp. 59-73.

Fraser, Nancy (1995b) "Pragmatism, Feminism, and the Linguistic Turn”, en Benhabib, Seyla; Butler, Judith; Cornell, Drucilla \& Fraser, Nancy, 1995, pp. 159-171.

Fraser, Nancy (1997) "Heterosexism, Misrecognition, and Capitalism: A Response to Judith Butler", Social Text 52/53, pp. 79-89.

Fuss, Diana, ed. (1991) Inside/out: lesbian theories, gay theories, New York, Routledge.

Garber, Marjorie; Hanssen, Beatrice; Walkowitz, Rebecca L. eds. (2000) The Return to Ethics, New York \& London, Routledge.

González Fisac, Jesús (2012) "Retorica y fenomenologia. Exterioridad y vulnerabilidad del campo del lenguaje”, en Soley-Beltrán, Patricia \& Sbasay, Leticia, eds. 2012b, pp: 169-194.

Grosz, Elizabeth (1995) Space, Time, and Perversion: The Politics of Bodies, St Leonards, NSW, Allen and Unwin.

Gubar, Susan (1998) “What Ails Feminist Criticism”, Critical Inquiry, 24(4), pp. 878-902.

Haverkamp, Anselm, ed. (1995) Deconstruction is/in America: A new sense of the political, New York University Press.

Hennessy, Rosemary (1995) “Queer Visibility in Commodity Culture”, Cultural Critique 29, pp. 31-76.

Honig, Bonnie, ed. (1995) Feminist Interpretations of Hannah Arendt, Penn State University Press.

Jenkins, Fiona (2010) "Disturbance, Provocation, and the Ethics of Non-Violence", Humanities Research 11(2), pp. 93-115.

Kirby, Vicki (2006) Judith Butler: Live Theory, London, Continuum.

Kruks, Sonia (2001) Retrieving Experience: Subjectivity and Recognition in Feminist Politics, Ithaca, NY, Cornell University Press.

Lloyd, Moya (1999) 'Performativity, Parody, Politics'. Theory, Culture and Society 16(2), pp. 195-213.

Lloyd, Moya (2005) Beyond Identity Politics: Feminism, Power and Politics, London, Sage. Lloyd, Moya (2007a) Judith Butler: From Norms to Politics, Cambridge, Polity Press.

Lloyd, Moya (2007b) 'Radical Democratic Activism and the Politics of Resignification'. Constellations 14(1), pp. 129-146. 
Lloyd, Moya (2008) "Towards a cultural politics of vulnerability: precarious lives and ingrievable deaths”, Carver, Terrell \& Chambers, Samuel A., eds., 2008, pp. 92-105.

Lloyd, Moya (2015a) “Introduction”, en Lloyd, Moya, ed., 2015b, pp.1-14.

Lloyd, Moya, ed. (2015b) Butler and Ethics, Edinburh University Press.

Loizidou, Elena (2007) Judith Butler: Ethics, Law, Politics, New York, Routledge-Cavendish.

Loizidou, Elena (2008a) "Butler and Life: Law, Sovereignty, and Power", en Carver, Terrell \& Chambers, Samuel A., eds., 2008, pp. 145-156.

Loizidou, Elena (2008b) "The Body Figural and Material in the Work of Judith Butler". Australian Feminist Law Journal 28(1), pp. 29-51.

Lovell, Terry (2003) "Resisting with Authority: Historical Specificity, Agency and the Performative Self", Theory, Culture and Society 20(1), pp. 1-17.

Magnus, Kathy Dow (2006) "The unaccountable subject: Judith Butler and the social conditions of intersubjective agency", Hypatia 21(2), pp. 81-103.

McNay, Lois (1999) "Subject, Psyche and Agency. The work of Judith Butler", Theory, Culture and Society, 16(2), pp. 175-193.

Mills, Catherine (2000) "Efficacy and Vulnerability: Judith Butler on Reiteration and Resistance”, Australian Feminist Studies 15(32), pp. 265-279.

Mills, Catherine (2007) "Normative Violence, Vulnerability and Responsibility", Differences: A Journal of Feminist Cultural Studies 18(2), pp. 134-156.

Nussbaum, Martha (1999) “The Professor of Parody", New Republic, (22 February 1999), $<$ http:www.tnr.com/archive/0299/022299/nussbaum022299.html>.

Pearce, Lynne (2004) The Rhetorics of Feminism: Readings in Contemporary Cultural Theory and the Popular Press, London, Routledge.

Pérez Navarro, Pablo (2012) "Parodias de la parodia en Martha Nussbaum y Celia Amorós”, en Soley-Beltrán, Patricia \& Sbasay, Leticia, eds., 2012b, pp. 27-58.

Rushing, Sara (2010) “Preparing for Politics: Judith Butler's ethical dispositions", Contemporary Political Theory, 9(3), pp. 284-303.

Salih, Sara (2002) Judith Butler, London, Routledge.

Schwartzman, Lisa H. (2002) "Hate Speech, Illocution, and Social Context: A Critique of Judith Butler”, Journal of Social Philosophy 33(3), pp. 421-441.

Searle, John (1969) Speech Acts. An essay in the philosophy of language, Cambridge University Press.

Searle, John (1989) "How Performatives work", Linguistics and Philosophy, 12(5), pp. 535-558.

Segal, Lynne (1994) Straight Sex: The Politics of Pleasure, London, Virago. 
Smith, Anna Marie (2001) “Words that Matter: Butler's Excitable Speech”, Constellations 8(3), pp. 390-399.

Soley-Beltrán, Patricia \& Sbasay, Leticia (2012a) “Introducción”, en Soley-Beltrán, Patricia y Sbasay, Leticia, eds., 2012b, pp. 9-26.

Soley-Beltrán \& Sbasay, Leticia, eds. (2012b) Judith Butler en disputa. Lecturas sobre la performatividad, Madrid, Egales.

Thiem, Annika (2008) Unbecoming subjects: Judith Butler, moral philosophy, and critical responsibility, New York, Fordham University Press.

Vasterling, Veronica (1999) "Butler's Sophisticated Constructivism: A Critical Assessment”, Hypatia 14(3), pp. 17-38.

Webster, Fiona (2000) "The Politics of Sex and Gender: Butler and Benhabib debate Subjectivity”, Hypatia 15(1), pp. 1-22.

Welton, Donn, ed. (1998) Body and Flesh: A Philosophical Reader, Oxford, Blackwell.

Weston, Kath (2002) Gender in Real Time: Power and Transience in a Visual Age, London \& New York, Routledge.

Zaharijevic, Adriana (2016) "In conversation with Judith Butler: Binds yet to be settled" Filozofija i Društvo, XXVII (1), pp. 105-114.

Zerilli, Linda (1995) “The Arendtian Body”, en Honig, Bonnie, ed., 1995, pp.167-194.

Zita, Jacquelyn N. (1992) "Male Lesbians and the Postmodernist Body”, Hypatia 7(4), pp. 106-127.

Zivi, Karen (2008) "Rights and the Politics of Performativity", en Carver, Terrell \& Chambers, Samuel A., eds., 2008, pp. 157-169. 